\title{
Kentron
}

Revue pluridisciplinaire du monde antique

$24 \mid 2008$

L'imaginaire utopique, de ses sources dans le monde grec à la Renaissance

\section{Bibliographie thématique}

\section{Corinne Jouanno}

\section{(2) OpenEdition}

Journals

Édition électronique

URL : http://journals.openedition.org/kentron/1596

DOI : 10.4000/kentron.1596

ISSN : 2264-1459

Éditeur

Presses universitaires de Caen

\section{Édition imprimée}

Date de publication : 1 décembre 2008

Pagination : 49-55

ISBN : 978-2-84133-322-6

ISSN : 0765-0590

Référence électronique

Corinne Jouanno, «Bibliographie thématique », Kentron [En ligne], 24 | 2008, mis en ligne le 13 mars 2018, consulté le 17 novembre 2020. URL : http://journals.openedition.org/kentron/1596 ; DOI : https://doi.org/10.4000/kentron.1596

\section{(c) (i) (9)}

Kentron is licensed under a Creative Commons Attribution-NonCommercial-NoDerivatives 3.0 International License. 


\section{L'IMAGINAIRE UTOPIQUE DANS LE MONDE GREC Bibliographie thématique}

\section{Études générales}

Baczko B. (1971), «Lumières et utopie. Problèmes de recherches », Annales ESC, 26, p. 355386.

Bасzко B. (1974), «L'utopie et l'idée de l'histoire-progrès ", Revue des Sciences humaines, 155, p. 473-491.

Bасzко B. (1978), Lumières de l'utopie, Paris, Payot.

BLOCH E. (1976-1982-1991), Le Principe espérance [1959], 3 vol., traduit de l'allemand par F. Wuilmart, Paris, Gallimard.

Bowman F.P. (1976), «Utopie, imagination, espérance: Northrop Frye, Ernst Bloch, Judith Schlanger », Littérature, 21, p. 10-19.

Cioranescu A. (1971), «Utopie, Cocagne et Âge d'or », Diogène, 75, p. 86-123.

Cioranescu A. (1972), L’Avenir du passé. Utopie et littérature, Paris, Gallimard.

Demersen G. (1981), "Cocagne, utopie populaire?», Revue belge de philologie et d'histoire, 59, p. 529-553.

De Gandillac M., Piron-Audard C. (dir.) (1978), Le Discours utopique (Entretiens, Centre culturel international de Cerisy-la-Salle, 23 juillet- ${ }^{\text {er }}$ août 1975), Paris, Union générale d'éditions $(10 / 18)$.

Dubois C.-G. (1968), Problèmes de l'utopie, Paris, Minard.

Lapouge G. (1973), Utopie et civilisations, Paris, Weber.

Manuel F.E. \& F.P. (1979), Utopian Thought in the Western World, Cambridge (MA), The Belknap Press of Harvard University Press.

Marouby C. (1990), Utopie et primitivisme. Essai sur l'imaginaire anthropologique à l'âge classique, Paris, Seuil.

Moreau P.-F. (1982), Le Récit utopique (droit naturel et roman de l'État), Paris, PUF.

Riot-Sarcey M., Bouchet T., Picon A. (éd.) (2002), Dictionnaire des utopies, Paris, Larousse.

Ruyer R. (1950), L'Utopie et les utopies, Paris, PUF.

SChlanger J. (1973), «Puissance et impuissance de l'imaginaire utopique », Diogène, 84, p. 327. 
Trousson R. (1974), «Utopie et roman utopique», Revue des Sciences humaines, 155, p. 367378.

Trousson R. (1975), Voyages aux Pays de Nulle-Part. Histoire littéraire de la pensée utopique, Bruxelles, Université de Bruxelles.

Trousson R. (1998), D’utopie et d'utopistes, Paris, L'Harmattan.

\section{Sur l'utopie en Grèce ancienne}

BALDry H.C. (1956), Ancient Utopias, Southampton, University of Southampton.

Beneitez Prudencio J.J. (2006), «Eutopia y edad de oro en la antigua Grecia. Un estudio sobre los albores del pensamiento utopico », Utopia and Utopianism, 1, p. 47-58.

Bertelli L. (1976), «Il modello della società rurale nell'utopia greca », Il pensiero politico, 9, p. $183-208$.

BERTELLI L. (1980), «L'utopia come specchio delle trasformazione sociali e politiche del mondo antico ", in Tra Grecia e Roma. Temi antichi e metodologie moderne, Rome, Istituto della enciclopedia italiana, p. 21-26.

Bertelli L. (1982), «L'utopia greca», in Storia delle idee politiche, economiche e sociali. 1. L'antichita classica, L. Firpo (éd.), Turin, Unione tipografico-editrice torinese, p. 463-581.

Bertelli L. (1985), «Schiavi in utopia », Studi storici, 4, p. 889-901.

BERTELLi L. (1986), «Esperienze urbanistiche nella costruzione della città ideale greca», in Utopie degli anni Ottanta: studi interdisciplinari sui temi, la storia, i progetti, G. Saccaro, A. O. Lewis (éd.), Rome, Gangemi, p. 291-311.

Bertelli L. (1987 a), "Genesi e vicenda dell'utopia greca », in Utopia e distopia, A. Colombo (éd.), Milan, Angeli, p. 221-252.

Bertelli L. (1987 b), «Itinerari dell'utopia greca : dalla città ideale alle isole felici », in La Città ideale nella tradizione classica e biblico-cristiana (Atti del convegno nazionale di studi, Torino, 2-4 Maggio 1985), R. Uglione (éd.), Turin, Regione Piemonte, p. 35-56.

Bertelli L. (1989), «I sogni della fame : dal mito all'utopia gastronomica », in Homo edens: regimi, miti e pratiche dell'alimentazione nella civiltà del Mediterraneo, O. Longo, P. Scarpi (éd.), Milan, Diapress, p. 103-114.

Bertelli L. (1990), "Geografia e paesaggio nell'utopia greca », in Scritti politici in onore di L. Firpo, 4 vol., S. Rota Ghibaudi, F. Barcia (éd.), Milan, F. Angeli, IV, p. 317-339.

Bertelli L. (1992), «L'utopia », in Lo spazio letterario della Grecia antica, vol. I, La produzione e la circolazione del testo, t. 1, La polis, Rome, Salerno editrice, p. 493-524.

Bichler R. (1984), «Zur historischen Beurteilung der griechischen Staatsutopie », Grazer Beiträge, 11, p. 179-206.

Bichler R. (1995), Von der Insel der Seligen zur Platons Staat. Geschichte der antiken Utopie, Vienne, Böhlau.

Bonner C. (1910), «Dionysiac Magic and the Greek Land of Cockaigne», TAPhA, 41, p. 175185. 
Braunert H. (1969), Utopia: Antworten griechischen Denkens auf die Herausforderung durch soziale Verhältnisse, Kiel, Hirt.

Carsana C., Cioccolo S., Schettino M.T. (2006), « Pensiero utopico e prassi politica nel mondo antico », Utopia and utopianism, 1, p. 9-46.

Dawson D. (1992), Cities of the Gods. Communist Utopias in Greek Thought, Oxford, Oxford University Press.

Ferguson J. (1975), Utopias of the Classical World, Londres, Thames \& Hudson.

Finley M.I. (1967), «Utopianism Ancient and Modern », in The Critical Spirit. Essays in honor of Herbert Marcuse, Boston, Beacon Press, p. 3-20.

Flashar H. (1974), Formen utopischen Denkens bei den Griechen, Innsbruck, Inst. f. Sprachwissenschaft d. Univ. Innsbruck.

Futre Pinheiro M.P. (2006), "Utopia and Utopias: A Study on a Literary Genre in Antiquity», in Authors, Authority, and Interpreters in the Ancient Novel. Essays in honor of Gareth L. Schmeling, E. P. Cueva, J. Alvares (éd.), Groningen, Barkhuis Publishing, p. 147-171.

Gernet L. (1933), «La cité future et le pays des morts », REG, 48, p. 293-310 [repris dans Anthropologie de la Grèce antique, Paris, F. Maspéro, 1968, p. 139-153].

Ghidini Tortorelli M. (1976 / 1978), «Miti e utopie nella Grecia antica », Annali dell'Istituto per gli studi storici, 5, p. 1-126.

GiANGRANDE L. (1976 / 1977), «Les utopies grecques », REA, 78 / 79, p. 120-128.

Giannini A. (1967), «Mito e utopia nella letteratura greca prima di Platone », Rendiconti dell'Istituto Lombardo, Classe di Lettere, 101, p. 101-132.

Günter R., Müller R. (1987), Sozialutopien der Antike, Leipzig, Edition Leipzig.

Hahn I. (1962), «Die soziale Utopie der Spätantike», Wissenschaftliche Zeitschrift der MartinLuther-Universität Halle-Wittenberg, gesch.-sprachwiss. Kl., 11, 1357-1361.

Herzog R. (1982), «Überlegungen zur griechischen Utopie: Gattungsgeschichte vor dem Prototyp der Gattung », in Utopieforschung. Interdisziplinäre Studien zur neuzeitlichen Utopie, 3 vol., W. Vosskamp (éd.), Stuttgart, J.B. Metzler, II, p. 1-2o.

Hunzinger C. (2002), «Boire en utopie: du pays de Cocagne comique aux utopies hellénistiques ", in Vin et santé en Grèce ancienne, J. Jouanna, L. Villard (éd.), Athènes, École française d'Athènes, p. 23-44.

Косн М. (1979), "Zur Utopie in der alten Welt», in Auf den Weg Gebracht. Idee und Wirklichkeit der Universität Konstanz (Festschrift K. G. Kiesinger), H. Sund, M. Timmermann (éd.), Constance, Univ. - Verl. Konstanz, p. 399-417.

Kytzler B. (1973), «Utopisches Denken und Handeln in der klassischen Antike», in Der utopische Roman, R. Villgradter, F. Krey (éd.), Darmstadt, Wissenschaftliche Buchgesellschaft, p. 45-68.

Lana I. (1949), "L'utopia di Ippodamo di Mileto », Rivista di filosofia, 40, p. 125-151 [repris dans Studi sul pensiero politico classico, Naples, Guida, 1973]. 
Polet A. (1947), Le Communisme dans la pensée grecque, Le Caire, Éditions de la Revue du Caire.

SAÏD S. (1986), "L'âge d'or des ethnographes grecs ", in Âge d'or et Apocalypse, B. Brugière,

R. Ellrodt (éd.), Paris, Publications de la Sorbonne, p. 91-10o.

SARTORi M. (1984), "Storia, "utopia” e mito nei primi libri della Bibliotheca historica di Diodoro Siculo », Athenaeum, 72, p. 492-536.

Sснмідт E.A. (1982), «Bukolik und Utopie. Zur Frage nach dem Utopischen in der antiken Hirtenpoesie », in Utopieforschung. Interdisziplinäre Studien zur neuzeitlichen Utopie, 3 vol., W. Vosskamp (éd.), Stuttgart, J.B. Metzler, II, p. 21-36.

VidAL-NAQuet P. (1972), «Esclavage et gynécocratie dans la tradition, le mythe, l'utopie», in Recherches sur les structures sociales dans l'Antiquité classique (Actes du colloque de Caen, 25-26 avril 1969), Paris, CNRS, p. 63-80.

Vogt J. (1971), «Die Sklaverei im utopischen Denken der Griechen », Rivista storicha dell'antichita, 1, p. 19-32.

\section{Sur Aristophane et la comédie grecque}

Auger D. (1979), «Le théâtre d'Aristophane: le mythe, l'utopie, les femmes », Les Cahiers de Fontenay, 17, p. 71-101.

Baldry H.C. (1953), «The Idler's Paradise in Attic Comedy», Greece and Rome, 22, p. 49-60.

Bertelli L. (1983), «L'utopia sulla scena. Aristofane e la parodia della città », Civiltà classica e cristiana, 4 , p. 215-261.

CARrière J.-C. (1979), Le Carnaval et la Politique: une introduction à la comédie grecque, suivie d'un choix de fragments, 2 éd., Paris, Les Belles Lettres (Annales littéraires de l'université de Besançon), p. 85-118 ( L'utopie comique»), p. 183-187 («Remarques sur l'utopie au $4^{\mathrm{e}}$ et au $3^{\mathrm{e}}$ s. »), p. 255-270 ("Trois descriptions du Pays de Cocagne»).

Dalfen J. (1975), «Politik und Utopie in den Vögeln des Aristophanes 451-638», Bolletino dell'Istituto di Filologia Greca di Padova, 2, p. 268-285.

Dobrov G.W. (éd.) (1997), The City as Comedy. Society and Representation in Athenian Drama, Chapell Hill, University of North Carolina Press, p. 1-132 ( "The theory and practice of utopia»).

Fauth W. (1973), « Kulinarisches und Utopisches in der griechischen Komödie », Wiener Studien, 86, p. 39-62.

Hubbard T.K. (1997), «Utopianism and the Sophistic City in Aristophanes », in Dobrov 1977, p. 23-50.

Konstan D. (1997), «The Greek Polis and Its Negations. Versions of Utopia in Aristophanes' Birds», in Dobrov 1997, p. 3-22.

LANGerbeck H. (1963), «Die Vorstellung vom Schlaraffenland in der alten attischen Komödie», Zeitschrift für Volkskunde, 59, p. 192-204. 
Pecirka J. (1963), «Aristophanes' Ekklesiazusae und die Utopien in der Krise der Polis », Wissenschaftliche Zeitschrift der Humboldt-Universität zu Berlin. Gesellschafts- und sprachwissenschaftliche Reihe, 12, p. 215-219.

Ruffel I. (2000), "The World Turned Upside Down: Utopia and Utopianism in the Fragments of Old Comedy", in The Rivals of Aristophanes. Studies in Athenian Old Comedy, D. Harvey, J. Wilkins (éd.), Londres, Duckworth - The Classical Press of Wales, p. 473506.

Schwinge E. (1977), "Aristophanes und die Utopie», Würzburger Jahrbücher für die Altertumswissenschaft, n.f. 3, p. 43-67.

Zimmermann B. (1983), "Utopisches und Utopie in den Komödien des Aristophanes ", Würzburger Jahrbücher für die Altertumswissenschaft, n.f. 9, p. 57-77 [repris (en traduction italienne) sous le titre «Nephelokokkygia. Riflessioni sull'utopia comica», in Carnevale e utopia nella Grecia antica, W. Rösler, B. Zimmermann (éd.), Bari, Levante, 1991, p. 53-101].

\section{Sur Platon et sa postérité}

Dougherty J. (1986), «The Geometries of Perfection: Atlantis, Utopia and the City of the Sun », Soundings, 69, p. 310-325.

Gadamer H.G. (1983), «Platos Denken in Utopien », Gymnasium, 90 ( ${ }^{\circ}$ 4-5), p. 434-455.

Gill C. (1976), «The Origin of the Atlantis Myth», Trivium, 11, p. 1-11.

Gill C. (1977), «The Genre of the Atlantis Story», Classical Philology, 72, p. 287-304.

Gill C. (1980), Plato: the Atlantis story. Timaeus 17-27. Critias, Bristol, Bristol Classical Press.

Lacks A. (1991), «L'utopie législative de Platon », Revue philosophique, 117, p. 417-428.

Lacroix J.-Y. (2007), L'Utopia de Thomas More et la tradition platonicienne. Fini et infini dans l'utopisme, Paris, Vrin.

Longo O. (1975 / 1976), «Strutture socio-economiche nella Repubblica di Platone: ideologia e utopia », Helikon, 15-16, p. 540-552.

Mattéi J.-F. (1996), Platon et le miroir du mythe. De l'âge d'or à l'Atlantide, Paris, PUF.

Neumann H. (1966 / 1967), «Plato’s Republic : Utopia or Dystopia?», Modern Schoolman, 44, p. 319-330.

Pradeau J.-F. (1997), Le Monde de la politique. Sur le récit atlante de Platon, Timée (17-27) et Critias, Sankt Augustin, Academia Verlag.

Pradeau J.-F. (2000), "L’Atlantide de Platon. L'utopie vraie», in Utopie. La quête de la société idéale, L. T. Sargent, R. Schaer (éd.), Paris, BnF - Fayard, p. 93-10o.

Quarta C. (1985), L'utopia platonica. Il progetto politico di un grande filosofo, Milan, F. Angeli. Romeri L., Veloso C. (2006), «Les origines de la cité chez Platon », Ktema, 31, p. 79-86. SAlin E. (1921), Platon und die griechische Utopie, Munich - Leipzig, Dunkler \& Humblot.

Schulte-Middelich B. (1982), «Möglichkeiten utopischen Denkens - Das Erbe Platons », in Alternative Welten, M. Pfister (éd.), Munich, W. Fink, p. 39-63. 
Vidal-Naquet P. (1991 a), «Athènes et l'Atlantide. Structure et signification d'un mythe platonicien ", in Le Chasseur noir. Formes de pensée et formes de société dans le monde grec, 3 éd., Paris, La Découverte, p. 335-36o.

Vidal-Naquet P. (1991 b), «Le mythe platonicien du Politique, les ambiguïtés de l'Âge d'or et de l'histoire», ibid., p. 361-380.

Vidal-Naquet P. (2005), L'Atlantide: petite histoire d'un mythe platonicien, Paris, Les Belles Lettres.

Welskopff E.C. (1963), «Zur Enstehung der Utopie bei Platon», Wissenschaftliche Zeitschrift der Humboldt-Universität zu Berlin. Gesellschafts- und sprachwissenschaftliche Reihe, 12, p. 229-235.

\section{Autres philosophes}

BALdry H.C. (1959), «Zeno's Ideal State », Journal of Hellenic Studies, 79, p. 3-15.

Bidez J. (1932), La Cité du monde et la cité du soleil chez les Stoüciens, Paris, Les Belles Lettres.

\section{Sur les utopies hellénistiques}

BAldAssarri M. (1973), «Intorno all'utopia di Giambulo », Rivista di filosofia neo-scolastica, 65, p. 303-333 et p. 471-487.

Brown W.E. (1955), «Some Hellenistic Utopias », The Classical Weekly, 48, p. 57-62.

Giangrande L. (1976), «Les utopies hellénistiques », Cahiers des études anciennes, 5, p. 17-33.

Holzberg N. (1996), «Utopias and Fantastic Travel: Euhemerus, Iambulus», in The Novel in the Ancient World, G. Schmeling (éd.), Leyde, Brill, p. 621-628.

Kytzler B. (1988), «Zum utopischen Roman der klassichen Antike », Groningen Colloquia on the Novel, 1, p. 7-16.

Lana I. (1951), «L’utopia di Teopompo », Paideia, 6, p. 3-22.

Mossé C. (1969), «Les utopies égalitaires à l'époque hellénistique », Revue historique, 241, p. 297-308.

Polet A. (1947), «Deux utopies hellénistiques: la Panchaïe d’Évhémère et la Cité du Soleil de Jambule», Bulletin of the Faculty of Arts, 9 / 1, p. 47-62.

Simon M. (1963), «Hellenistische Märchenutopien », Wissenschaftliche Zeitschrift der Humboldt-Universität zu Berlin. Gesellschafts- und sprachwissenschaftliche Reihe, 12, p. 237-243.

Vernière Y. (1988), «Îles mythiques chez Diodore de Sicile», in Peuples et pays mythiques (Actes du V $\mathrm{V}^{\mathrm{e}}$ colloque du Centre de recherches mythologiques de l'université de Paris X, Chantilly, 18-20 septembre 1986), F. Jouan, B. Deforge (éd.), Paris, Les Belles Lettres, p. 159167.

Winiarczy K M. (1997), «Das Werk des Jambulos. Forschungsgeschichte (1550-1988) und Interpretationsversuch ", RhMus, 140, p. 128-153.

Winston D. (1956), Jambulus. A Literary Study in Greek Utopianism, diss. Columbia University. 


\section{Sur Lucien et sa postérité}

Dorsch T.S. (1967), «Sir Thomas More and Lucian : an Interpretation of Utopia », Archiv für das Studium der neueren Sprachen und Literaturen, 203, p. 349-363.

FAUTH W. (1979), «Utopische Inseln in den Wahren Geschichten des Lukians », Gymnasium, 86, p. 39-58.

Fusillo M. (1988), « Le miroir de la Lune. L'Histoire vraie de Lucien, de la satire à l'utopie», Poétique, 73, p. 109-135.

Lauvergnat-Gagnière C. (1978), "Rabelais lecteur de Lucien de Samosate », Cahiers de l'Association internationale des études françaises, 30, p. 71-86.

Nesselrath H.G. (1993), «Utopie-Parodie in Lukians Wahren Geschichten », in Literaturparodie in Antike und Mittelalter, W. Ax, R.F. Glei (éd.), Trèves, Wissenschaftlicher Verlag Trier, p. 41-56. 